\title{
The mass-loss return from evolved stars to the Large Magellanic Cloud
}

\section{Dust properties for carbon-rich asymptotic giant branch stars}

\author{
S. Srinivasan ${ }^{1}$, B. A. Sargent ${ }^{2}$, M. Matsuura ${ }^{3,4}$, M. Meixner ${ }^{2}$, F. Kemper ${ }^{5}$, A. G. G. M. Tielens ${ }^{6}$, K. Volk ${ }^{2}$, \\ A. K. Speck ${ }^{7}$, P. M. Woods ${ }^{5}$, K. Gordon ${ }^{2}$, M. Marengo ${ }^{8}$, and G. C. Sloan ${ }^{9}$ \\ ${ }^{1}$ Institut d'Astrophysique de Paris, 98bis boulevard Arago, 75014 Paris, France \\ e-mail: srinivas@iap.fr \\ 2 Space Telescope Science Institute, 3700 San Martin Drive, Baltimore, MD 21218, USA \\ 3 UCL-Institute of Origins, Astrophysics Group, Department of Physics and Astronomy, University College London, Gower Street, \\ London WC1E 6BT, UK \\ ${ }^{4}$ UCL-Institute of Origins, Mullard Space Science Laboratory, University College London, Holmbury St. Mary, Dorking, \\ Surrey RH5 6NT, UK \\ 5 Jodrell Bank Centre for Astrophysics, School of Physics \& Astronomy, University of Manchester, Manchester, M13 9PL, UK \\ ${ }^{6}$ NASA Ames Research Center, SOFIA Office, MS 211-3, Moffet Field, CA 94035, USA \\ 7 Department of Physics and Astronomy, University of Missouri, Columbia, MO 65211, USA \\ 8 Department of Physics and Astronomy, Iowa State University, Ames, IA 50010, USA \\ 9 Center for Radiophysics and Space Research, Cornell University, 222 Space Sciences Building, Ithaca, NY 14853, USA
}

Received 14 May 2010 / Accepted 12 September 2010

\section{ABSTRACT}

\begin{abstract}
We present a radiative transfer model for the circumstellar dust shell around a Large Magellanic Cloud (LMC) long-period variable (LPV) previously studied as part of the Optical Gravitational Lensing Experiment (OGLE) survey of the LMC. OGLE LMC LPV 28579 (SAGE J051306.40-690946.3) is a carbon-rich asymptotic giant branch (AGB) star for which we have Spitzer broadband photometry and spectra from the SAGE and SAGE-Spec programs along with broadband UBVIJHK shotometry. By modeling this source, we obtain a baseline set of dust properties to be used in the construction of a grid of models for carbon stars. We reproduce the spectral energy distribution of the source using a mixture of amorphous carbon and silicon carbide with $15 \%$ SiC by mass. The grain sizes are distributed according to the $\mathrm{KMH}$ model, with $\gamma=3.5, a_{\min }=0.01 \mu \mathrm{m}$ and $a_{0}=1.0 \mu \mathrm{m}$. The best-fit model produces an optical depth of 0.28 for the dust shell at the peak of the $\operatorname{SiC}$ feature $(11.3 \mu \mathrm{m})$, with an inner radius of about $1430 R_{\odot}$ or 4.4 times the stellar radius. The temperature at this inner radius is $1310 \mathrm{~K}$. Assuming an expansion velocity of $10 \mathrm{~km} \mathrm{~s}^{-1}$, we obtain a dust mass-loss rate of $2.5 \times 10^{-9} M_{\odot} \mathrm{yr}^{-1}$. We calculate a $15 \%$ variation in this mass-loss rate by testing the sensitivity of the fit to variation in the input parameters. We also present a simple model for the molecular gas in the extended atmosphere that could give rise to the $13.7 \mu \mathrm{m}$ feature seen in the spectrum. We find that a combination of $\mathrm{CO}$ and $\mathrm{C}_{2} \mathrm{H}_{2}$ gas at an excitation temperature of about $1000 \mathrm{~K}$ and column densities of $3 \times 10^{21} \mathrm{~cm}^{-2}$ and $10^{19} \mathrm{~cm}^{-2}$ respectively are able to reproduce the observations. Given that the excitation temperature is close to the temperature of the dust at the inner radius, most of the molecular contribution probably arises from this region. The luminosity corresponding to the first epoch of SAGE observations is $6580 L_{\odot}$. For an effective temperature of about $3000 \mathrm{~K}$, this implies a stellar mass of 1.5-2 $M_{\odot}$ and an age of 1-2.5 Gyr for OGLE LMC LPV 28579. We calculate a gas mass-loss rate of $5.0 \times 10^{-7} M_{\odot} \mathrm{yr}^{-1}$ assuming a gas:dust ratio of 200 . This number is comparable to the gas mass-loss rates estimated from the period, color and $8 \mu \mathrm{m}$ flux of the source.
\end{abstract}

Key words. stars: AGB and post-AGB - stars: carbon - circumstellar matter - stars: mass-loss - Magellanic Clouds stars: individual: OGLE LMC LPV 28579

\section{Introduction}

The asymptotic giant branch (AGB) is the final stage in the evolution of low- and intermediate-mass stars $\left(0.8\right.$ to $\left.8 M_{\odot}\right)$. Products of the nuclear reactions in the AGB star interiors are brought to the outer regions where they form molecules and, farther from the star, dust grains. Carbon-rich (C-rich) AGB stars are created by the process of the third dredge-up (Iben \& Renzini 1983). Slow winds, with typical expansion velocities of $10 \mathrm{~km} \mathrm{~s}^{-1}$, feed the processed material (gas and dust) back into the surrounding interstellar medium (ISM) at rates up to $10^{-5}-10^{-4} M_{\odot} \mathrm{yr}^{-1}$, where it is eventually locked into newly forming stars. The large numbers of AGB stars makes them significant contributors to the chemical evolution of galaxies. In particular, C-rich AGB stars are the among the major sources of carbon atoms (Dwek 1998) and carbonaceous dust grains (Dwek 1998; Matsuura et al. 2009) in a galaxy. It is therefore crucial to determine the composition of AGB star dust and the total injection rate of this dust into the ISM.

The study of Galactic carbon stars is complicated due to the high (and often unknown) line-of-sight extinction towards these sources. Despite this fact, a lot of information has been 
gained about the circumstellar dust around these stars. The Large Magellanic Cloud offers an exceptional environment for the study of the mass-loss return and enrichment process by AGB stars. Recent near-infrared (NIR) and mid-infrared (MIR) surveys (e.g., 2MASS, Skrutskie et al. (2006); DENIS, Epchtein et al. (1994) and SAGE, Meixner et al. (2006)) have revealed tens of thousands of AGB star candidates in Large Magellanic Cloud (LMC). The SAGE (Meixner et al. 2006) survey has observed the LMC in the Spitzer Space Telescope (Spitzer; Gehrz et al. 2007) Infrared Array Camera (IRAC; 3.6, 4.5, 5.8 and $8.0 \mu \mathrm{m})$ and Multiband Imaging Photometer for Spitzer (MIPS; 24, 70 and $160 \mu \mathrm{m}$ ) bands. Blum et al. (2006) and Srinivasan et al. (2009) (hereafter, Paper I) used SAGE photometry to identify about 7000 carbon-rich AGB star candidates in the LMC. Follow-up spectroscopy using the Infrared Spectrograph (IRS) on Spitzer has also been performed on selected SAGE targets as part of the SAGE-Spec (Kemper et al. 2010) program.

Detailed radiative transfer (RT) studies conducted on Galactic and extragalactic carbon stars in the past have helped identify many dust species as possible constituents of their circumstellar envelopes. Here we concentrate on two species that significantly affect the near- and mid-IR photometry and spectroscopy, carbon dust (amorphous carbon or graphite) and silicon carbide ( $\alpha$ or $\beta$ type). IRC +10216 (Neugebauer \& Leighton 1969 ) is the best-studied carbon star in the galaxy, and a majority of early studies involved the modeling of its circumstellar shell (e.g., Jones \& Merrill 1976; Fazio et al. 1980). Rowan-Robinson \& Harris (1983) used their RT code to fit the circumstellar envelopes (CSEs) of 44 Galactic carbon stars and found that amorphous carbon dust (hereafter AmC) produced better fits than graphite. A similar result was obtained by Martin \& Rogers (1987). Skinner \& Whitmore (1988) found that the strength of the $\mathrm{SiC}$ feature in carbon stars was inversely proportional to the total mass-loss rate which they explained as being due to a decrease in the $\mathrm{SiC} / \mathrm{AmC}$ ratio with increasing mass-loss rate, thus decreasing the prominence of the $\mathrm{SiC}$ feature in comparison to the AmC continuum. This decrease in $\mathrm{SiC} / \mathrm{AmC}$ ratio with increasing mass loss was verified by Chan \& Kwok (1990) and explained as being due to carbon star evolution. Similar trends were also reported by Lorenz-Martins \& Lefèvre (1993, 1994) and by Groenewegen (1995). Groenewegen (1995), Speck et al. (1997) and Blanco et al. (1998) compared the results of models using $\alpha$ - and $\beta$-SiC, finding that the dust containing $\alpha$-SiC was able to reproduce the spectra of most of their sources. Bressan et al. (1998) included a treatment of AGB dust shells in their stellar population models, and they generated isochrones for comparison with IRAS data for Miras and OH/IR stars. van Loon et al. (1999) performed RT modeling on the ISO spectroscopy of LMC AGB stars to identify the chemistry of the circumstellar dust and calculated their mass-loss rates and luminosities. Suh (2000) obtained empirical opacity functions for amorphous carbon dust based on their RT modeling of IR spectra as well as laboratory-measured optical data. Groenewegen (2006) presented synthetic photometry for O-rich and C-rich AGB stars from the results of RT models that spanned the relevant range of stellar and dust shell parameters seen in Galactic AGB stars. In particular, they considered two kinds of dust species for carbon star dust shells: AmC and a mixture of AmC and $15 \% \alpha-\mathrm{SiC}$ by mass. The theoretical work of Mattsson et al. (2008) and Wachter et al. (2008) showed that the mass-loss rates of carbon stars may not be sensitive to metallicity. Numerous recent studies using Spitzer spectra of AGB stars in low-metallicity Local Group galaxies (e.g., Sloan et al. 2006; Zijlstra et al. 2006; Lagadec et al. 2007; Matsuura et al. 2007; Sloan et al. 2008) seem to support this claim. Similar results were obtained by Groenewegen et al. (2007), who found in their modeling of the IRS spectra of 60 carbon stars in the Magellanic Clouds that the trend of mass-loss rates with period of luminosity was comparable to that of Galactic sources. Groenewegen et al. (2009) extended this study to a larger sample, including 110 carbon stars, and compared their mass-loss rates and luminosities with those predicted by evolutionary models as well as dynamical wind models.

Our aim is to develop a grid of RT models for dust shells around carbon stars so that we may fit the SAGE photometry of carbon star candidates and derive their mass-loss rates. These mass-loss rates will enable the calculation of the total carbonstar mass-loss return to the LMC. As a first step, in this paper, we determine a representative set of dust properties for LMC C-rich AGB stars to be used as input to the modeling. We present a 2Dust radiative transfer model for the circumstellar shell around the variable carbon star OGLE LMC LPV 28579 (SAGE J051306.40-690946.3) with the primary goal of deriving the dust properties with a physically realistic model of the source, as constrained by available data. We have also used this approach in Sargent et al. 2010 (in press) for the O-rich AGB stars and red supergiants. This paper is organized as follows. We describe the observational data for OGLE LMC LPV 28579 (hereafter LPV 28579) from various studies in Sect. 2. In Sect. 3, we provide details of our radiative transfer model for the circumstellar dust, as well as a simple model for the molecular features observed in the spectrum. We discuss the results of the models in Sect. 4.

\section{Observations}

The choice of LPV 28579 for this study was motivated by the availability of both SAGE photometry and SAGE-Spec spectroscopic data. The SAGE-Spec program selected a bright subsample of the SAGE AGB candidates for good quality spectra. This requirement means that the spectroscopic sample is biased towards redder colors, and that LPV 28579 is redder than most carbon stars in the LMC. Nevertheless, it is only moderately optically thick, and exhibits features found in typical carbon stars. Its spectrum (see Sect. 2.3 for details) shows the overall continuum emission from carbon dust as well as a significant $11.3 \mu \mathrm{m}$ feature due to $\mathrm{SiC}$, which makes it a good testbed for the dust properties around LMC carbon stars. Groenewegen (2004) classified the star as an obscured AGB candidate, while in Paper I we labelled it an "extreme" AGB star based on the Blum et al. (2006) selection criterion $(J-[3.6]>3.1 \mathrm{mag})$. Examination of the SAGE-Spec spectrum (Sect. 2.3) shows the $11.3 \mu \mathrm{m}$ silicon carbide feature to be in emission. Moreover, results from the present modeling work (Sect. 3.1.5) suggest at best a moderate $11.3 \mu \mathrm{m}$ optical depth. Based on this information, LPV 28579 would not be considered an extreme carbon star (Speck et al. 2009).

Photometry for LPV 28579 is available from many recent LMC surveys (e.g., Zebrun et al. 2001; Epchtein et al. 1999; Cutri et al. 2003; Meixner et al. 2006; Kato et al. 2007), enabling us to constrain its spectral energy distribution (SED). We have combined the SAGE data with photometry from the optical Magellanic Clouds Photometric Survey (MCPS; Zaritsky et al. 1997) as well as the 2 micron All Sky Survey (2MASS, Skrutskie et al. 2006). We discuss the optical and NIR variability observations in Sect. 2.1, the SAGE photometry in Sect. 2.2 and the SAGE-Spec data in Sect. 2.3. In Fig. 1, we show the full range of photometry and spectroscopy values measured to illustrate the 


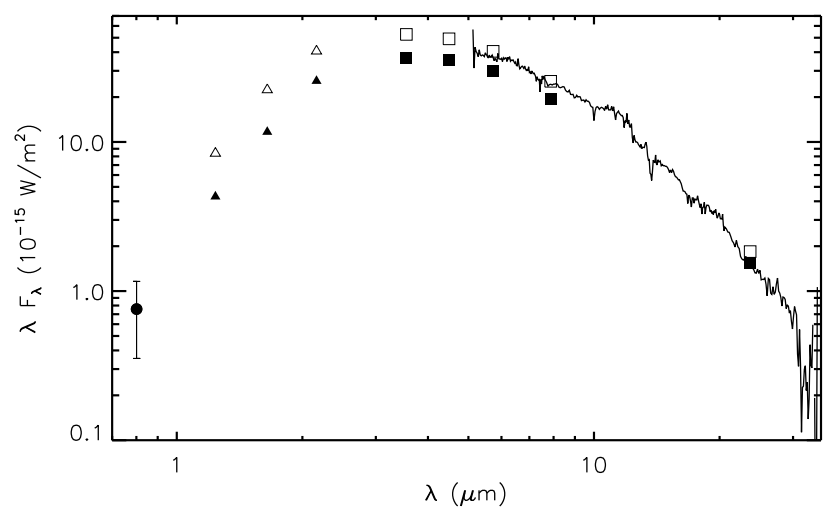

Fig. 1. A compilation of photometric and spectroscopic information for LPV 28579. The solid circle and error bar show the mean OGLE $I$ band flux and its range of variation respectively. The 2MASS (open triangles), IRSF (filled triangles) and SAGE (Epoch 1: open squares, epoch 2: filled squares) fluxes are also shown. The solid curve is the SAGE-Spec IRS spectrum.

source variability. In this paper, we only model the SAGE Epoch 1 photometry, using the optical and NIR data along with the IRS spectrum to constrain the shape of the resulting SED.

\subsection{Variability in optical and NIR data}

LPV 28579 was observed as part of the Optical Gravitational Lensing Experiment (OGLE-II; Zebrun et al. 2001) survey of the Magellanic Clouds. The $I$ band light curve is available as part of the OGLE-II variable star catalog (Szymański 2005) as well as the OGLE-III list of long-period variables (LPVs) as described in Soszyński et al. (2009). Based on its light curve, LPV 28579 was classified as a Mira-type LPV. Ita et al. (2004) and Groenewegen (2004) crossmatched the OGLE-II data with the IRSF LMC Survey (Kato et al. 2007), DENIS (Epchtein et al. 1999) and 2MASS All-Sky Release (Cutri et al. 2003) NIR catalogs and fit the light curves to obtain variability periods and amplitudes. Ita et al. (2004) and Groenewegen (2004) calculated the period of variation to be $361 \mathrm{~d}$ and $358.6 \pm 0.136 \mathrm{~d}$ respectively. Ita et al. (2004) also reported a mean I magnitude of $17.483 \mathrm{mag}$ and a peak-to-peak amplitude of 1.865 mag. The OGLE-III catalog lists the mean magnitude as 17.586 mag. The light curve available from the OGLE-III website ${ }^{1}$ shows that LPV 28579 is multi-periodic, with a primary period of $356.2 \mathrm{~d}$ (peak-to-peak amplitude $1.587 \mathrm{mag}$ ) modulated by a slow variation of $5747 \mathrm{~d}$ (peak-to-peak amplitude $0.784 \mathrm{mag}$ ). Like many of our extreme AGB candidates identified in Paper I, LPV 28579 is absent from the Fraser et al. (2008) MACHO catalog of LMC LPVs. This is most likely due to the moderately high circumstellar extinction at optical wavelengths. The primary period for LPV 28579 is very close to those of the sources in the "one year artifact" list, but the culling of these artifacts in Fraser et al. (2008) was done in a manner as to avoid accidental removal of genuine sources. Based on its brightness $\left(K_{\mathrm{s}}=10.9 \mathrm{mag}\right)$ and period, LPV 28579 would probably fall on period sequence 1 , the sequence found by Wood et al. (1999) to be predominantly populated by Miras in their fundamental mode of pulsation. While we do not have $J H K_{\mathrm{s}}$ light

\footnotetext{
${ }^{1}$ http://ogledb.astrouw.edu.pl/ ogle/CVS/
}

curves, the 2MASS and IRSF photometry for LPV 28579 capture some of the NIR flux variation ${ }^{2}$ (Fig. 1).

The optical properties of LPV 28579 are fairly normal for LMC carbon stars - the pulsation period of $\sim 1 \mathrm{yr}$ is average for typical LMC carbon Miras. It has a relatively small amplitude in comparison to other carbon Miras in the OGLE-III catalog (amplitudes ranging from $0.8 \mathrm{mag}$ to $5.7 \mathrm{mag}$ ), and this points to a somewhat hotter central star than a very cool one. The moderate amplitude combined with the $\sim 1 \mathrm{yr}$ period make OGLE LMC LPV 28579 a typical member of the LMC carbon Mira family. Furthermore, while LPV 28579 has very red colors $(J-K=3.27 \mathrm{mag})$, it is nowhere nearly as red as, e.g., IRC+10216 $(J-K=6.15$ mag; Whitelock et al. 2006).

\subsection{SAGE photometry}

The SAGE survey (Meixner et al. 2006) imaged a $\sim 7^{\circ} \times 7^{\circ}$ area of the Large Magellanic Cloud (LMC) with the Spitzer Infrared Array Camera (IRAC; Fazio et al. 2004, 3.6, 4.5, 5.8 and $8.0 \mu \mathrm{m}$ ) and Multiband Imaging Photometer (MIPS; Rieke et al. 2004, 24, 70 and $160 \mu \mathrm{m})$. Two epochs of observations separated by three months were obtained to constrain source variability. IRAC and MIPS epoch 1 and epoch 2 archive photometry for LPV 28579 is available as part of SAGE data delivered to the Spitzer Science Center ${ }^{3}$ (SSC). By comparing data from both epochs of SAGE, Vijh et al. (2009) identified infrared variable sources in the LMC of which $\sim 81 \%$ are AGB candidates. LPV 28579 is one of the 2000 SAGE variables discovered by Vijh et al. (2009). Figure 1 shows both epochs of SAGE data. In this paper, we model the Epoch 1 data (the brighter set of fluxes in Fig. 1) and use the Epoch 2 photometry only to estimate the range of variability of the source (see Sect. 3.1.1).

\subsection{SAGE-Spec data}

Spectroscopic follow-up observations to SAGE using the Infrared Spectrograph (IRS) aboard Spitzer were performed as part of the SAGE-Spectroscopy survey (Kemper et al. 2010). The spectra were reduced using techniques described in the SAGE-Spec Data Delivery Handbook ${ }^{4}$ (Woods et al. 2009) and summarized briefly as follows. LPV 28579 was observed in both spectral orders of the Short-Low (SL; 5.2-14.3 $\mu \mathrm{m}, R \sim$ 60-120) and Long-Low (LL; 14.3-13.7 $\mu \mathrm{m}, R \sim 60-120)$ instruments on board Spitzer at two nod positions. Data from the S15.3 and S17.2 pipelines for SL and LL, respectively, were obtained from the SSC for LPV 28579 (AOR \# 22415360). SL sky subtraction was performed by subtracting the observations in one order from the other while keeping the nod position fixed, while LL sky subtraction involved subtracting one nod position from the other while keeping the order fixed. Bad pixels on the detector arrays were replaced with values determined by comparing to the local point-spread function ${ }^{5}$.

\footnotetext{
2 The difference in $K_{\mathrm{s}}$ magnitude between the two epochs is 0.5 mag. Comparing this result to Fig. 3 of Whitelock et al. (2003), which shows the $K$-band pulsation amplitude as a function of period, we find that the amplitude corresponding to the primary period for LPV 28579 is $\sim 0.4-0.8$ mag.

3 Data version S13 available on the SSC website, http://ssc. spitzer.caltech.edu/legacy/sagehistory.html

${ }^{4}$ Handbook version 1 (30 July 2009) available on the SSC website, http://data.spitzer. caltech. edu/popular/sage-spec/

${ }^{5}$ For details, please refer to the IRS Data Handbook:

http://ssc. spitzer. caltech. edu/IRS/dh/dh32 . pdf.
} 
The width of the apertures used for point source signal extraction increased in proportion to the wavelength; this involved using the profile, ridge and extract modules ${ }^{6}$. Flux densities were obtained by calibrating the raw signal extractions using observations of the standard stars HR 6348 (K0 III), HD 166780 (K4 III) and HD 173511 (K5 III). While all three standard stars were used for LL, only the first was used for SL. Where available, the mean flux from multiple data collection events (DCEs) for a single module/order/nod was computed, with the standard deviation from the mean providing a measure of the corresponding uncertainty. Spikes not eliminated by replacement of bad pixel values were effectively removed by replacing any uncertainty larger than the average in the local wavelength range by the uncertainty from the nod position closer to the local average. Bonus-order spectra (corresponding to a small segment of the first-order spectrum obtained by the second-order slit) were averaged with spectra from the second and first orders over wavelengths they shared in common.

The IRS spectrum for LPV 28579 (Fig. 1) shows a broad $11.3 \mu \mathrm{m} \mathrm{SiC}$ emission feature combined with a featureless continuum characteristic of carbonaceous dust. LPV 28579 does not show any particular signs of the 30 micron feature which is strong in the more extreme Galactic carbon stars (Hrivnak et al. 2009) and is also seen in very red LMC carbon stars in the SAGE-Spec sample. There is also a $13.7 \mu \mathrm{m} \mathrm{C}_{2} \mathrm{H}_{2}$ feature. The strength of this feature cannot be reproduced by a model photosphere alone, pointing to its origin in the extended atmosphere and circumstellar shell (see Matsuura et al. 2006). We model both the circumstellar dust as well as the molecular gas in the extended atmosphere in Sect. 3. We note two trends at longer wavelengths: there is a step-down in the flux at $\sim 20.5 \mu \mathrm{m}$, and the flux errors for the entire Long-Low 1st order (LL1) wavelength range are on average very large. These are suggestive of sky-subtraction issues, so we checked the raw data for LL1. We find that there is a noticeable amount of extended emission in LL1 due to an extremely red source creeping into the slit, which affects the background subtraction.

The optical, NIR and MIR photometry as well as MIR spectroscopy described above are incorporated into Fig. 1. The twoepoch photometry shows that there is no significant change in the shape of the MIR SED. We have taken advantage of this fact and scaled the SAGE-Spec spectrum down to the SAGE Epoch 1 $5.8 \mu \mathrm{m}$ flux to enable easy comparison to photometry. This scaling changes the flux predicted for the spectrum in the $5.8 \mu \mathrm{m}$ band by $\sim 5 \%$.

\section{Analysis}

\subsection{Dust radiative transfer models}

We model the emission from LPV 28579 using the twodimensional radiative transfer code 2Dust (Ueta \& Meixner 2003). For simplicity, we assume a spherical geometry for this study. 2Dust is capable of radiative transfer of axisymmetric systems. This may be needed for describing the most evolved AGB stars as well as their post-AGB counterparts in the model grid. Although AGB mass loss appears to be largely spherical, the successors to these objects, the post-AGB stars, are expected to be bipolar (as are their Galactic counterparts). The dust around post-AGB stars is similar to those around the most evolved AGB stars, therefore we allow for the model grid to cover postAGB stars and pre-planetary nebulae (PPNe).

\footnotetext{
${ }^{6}$ These modules are available in SPICE, the Spitzer IRS Custom Extraction package.
}

Table 1. 2Dust Parameters For LPV 28579.

\begin{tabular}{ll}
\hline \hline Photosphere model $^{a}$ & \\
$T_{\text {eff }}(\mathrm{K})$ & 3000 \\
$L_{*}\left(L_{\odot}\right)$ & $6580(6150-7010)^{b}($ Epoch 1$)$ \\
& $4810($ Epoch 2$)$ \\
$R_{*}\left(R_{\odot}\right)$ & $325($ Epoch 1$)$ \\
C/O & 1.3
\end{tabular}

Dust shell properties

$\begin{array}{ll}R_{\text {in }}\left(R_{*}\right) & 4.4(4.0-4.8) \\ R_{\text {out }}\left(R_{\text {in }}\right) & 1000 \\ \text { Density profile } & \rho(r) \sim r^{-2} \\ v_{\text {exp }}\left(\mathrm{km} \mathrm{s}^{-1}\right) & 10 \\ \text { Gas:dust ratio } & 200\end{array}$

\section{Dust grain properties}

$\begin{array}{ll}\text { Species } & \mathrm{AmC}^{c}+\mathrm{SiC}^{d} \\ \text { SiC fraction } & 12 \%(10 \%-16 \%) \\ \tau(11 \mu \mathrm{m}) & 0.27(0.25-0.275) \\ \text { Size distribution } & \mathrm{KMH}^{e} \\ & a_{\min }(\mu \mathrm{m})=0.01(<0.1) \\ & a_{0}(\mu \mathrm{m})=1(0.75-1.3) \\ & \gamma=3.5\end{array}$

Mass-loss rate and dust temperature

$$
\begin{array}{ll}
\dot{M}_{\mathrm{d}}\left(\times 10^{-9} M_{\odot} \mathrm{yr}^{-1}\right) & 2.5(2.4-2.9) \\
\dot{M}_{\mathrm{g}}\left(\times 10^{-7} M_{\odot} \mathrm{yr}^{-1}\right) & 5.0(4.8-5.8) \\
T_{\text {in }}(\mathrm{K}) & 1310(1260-1380) \\
\hline
\end{array}
$$

Notes. (a) Photosphere model from Gautschy-Loidl et al. (2004). (b) Uncertainty in parameter value (see Sect. 3.1.5 for details). (c) Amorphous carbon grains, $\rho=1.8 \mathrm{~g} \mathrm{~cm}^{-3}$, optical constants from Zubko et al. (1996). (d) SiC grains, $\rho=3.22 \mathrm{~g} \mathrm{~cm}^{-3}$, optical constants from Pégourié (1988). ${ }^{(e)}$ Size distribution from Kim et al. (1994): $n(a) \sim a^{-\gamma} \exp \left(-a / a_{0}\right)$ with $a>a_{\min }$.

The goal of the present study is to extract a set of dust properties for LPV 28579 that will also provide a range of reasonable properties for our entire C-rich AGB sample. As input, 2Dust needs information about the central star (effective temperature, size, distance and SED), the shell geometry (inner and outer radius, density variation), and the dust grains (species, optical depth at a reference wavelength, grain size distribution). Table 1 summarizes the model parameters that define the star+shell system. Several of these parameters are very sensitive to the modeling process and for these we have shown a best fit parameter, as well as a range of acceptable fits, in the table. Other parameters are adopted based on reasonable assumptions because of limited choices, ancillary data or because the modeling process is not very sensitive to their choice. We describe the selection of these parameters and the estimation of the parameter range in the subsections below.

\subsubsection{Central star}

We model the central star using the carbon star model photospheres of Gautschy-Loidl et al. (2004), placing it at a distance of $50 \mathrm{kpc}$ (LMC distance; see, e.g., Feast 1999; van Leeuwen et al. 2007). We prefer these model spectra over a simple blackbody, as the inclusion of molecular absorption changes the photospheric features in the optical and NIR, which in turn affects the output spectrum (a 15-20\% difference in IRAC fluxes between the $3000 \mathrm{~K}$ blackbody and the $3000 \mathrm{~K}$ 
model photosphere discussed below). The Gautschy-Loidl et al. (2004) set includes 29 solar-mass, solar-metallicity models. As photospheric molecular features are sensitive to metallicity (e.g., Matsuura et al. 2002), we may not be able to faithfully reproduce their strengths in LMC stars $\left(Z / Z_{\odot} \sim 0.3-0.5\right.$ for the LMC; Westerlund 1997), but such differences for LPV 28579 would be overwhelmed by emission from its optically thick dust shell. The near-IR photometry suffers from circumstellar extinction, making it difficult to constrain the effective temperature and surface gravity. Therefore, we are only able to find a suitable model by using the source luminosity as a constraint, which we estimate to be $(7690 \pm 400) L_{\odot}$ for the source using the Epoch 1 fluxes. Two models have luminosities of $6090 \mathrm{~L}_{\odot}$, within $20 \%$ of our estimate for the star. They have effective stellar temperatures of $3000 \mathrm{~K}$ and $3100 \mathrm{~K}$, and $\log g$ values of -0.55 and -0.49 respectively. To model LPV 28579, we use the $3000 \mathrm{~K}$ photosphere. Keeping all other parameters fixed, we found that there is very little difference in the near- and mid-IR output SEDs if we picked the $3100 \mathrm{~K}$ model instead. The model photosphere has a $\mathrm{C} / \mathrm{O}$ ratio of 1.3 . While this value is typical for Galactic Miras, the $\mathrm{C} / \mathrm{O}$ ratio may be higher for Magellanic Cloud carbon stars (see, e.g., Matsuura et al. 2005; Stanghellini et al. 2005), which would change the optical depth due to increased molecular opacity. However, since we are limited by the small number of model photospheres available, we ignore these effects.

\subsubsection{Circumstellar shell geometry}

We make the simplifying assumption of spherical symmetry as we are more interested in constraining the dust properties in this study. We also assume a constant mass-loss rate and outflow velocity $v_{\text {exp }}$, leading to an inverse-square density distribution in the shell. The lower metallicity of the LMC suggests lower $v_{\text {exp }}$ values for the O-rich AGB outflows compared to their Galactic counterparts, but the outflow velocities of carbon stars are less sensitive to metallicity (Habing 1996). Wachter et al. (2008) compute carbon-star dynamical wind models for subsolar metallicities. They find that the outflow velocities of solar metallicity models are about twice that of the LMC. These theoretical predictions of lower outflow velocities at lower metallicity are supported by the observations of Lagadec et al. (2010). If the expansion velocity is assumed to be independent of distance in the shell, it has no effect on the shape of the output SED and cannot, therefore, be constrained by performing radiative transfer. For simplicity, we assume a value of $v_{\text {exp }}=10 \mathrm{~km} \mathrm{~s}^{-1}$, which is in agreement with observations of Galactic C-rich AGB stars of similar periods (see Fig. 4 of Marigo et al. 2008). The massloss rate depends linearly on $v_{\text {exp }}$ (see Eq. (2)) for our simplifying set of assumptions, therefore the calculated rate will be lower if the outflow velocity is lower than the Galactic value.

Radiative transfer codes like DUSTY (Nenkova et al. 2000) accept the dust temperature at the inner radius as input and calculate the inner radius on execution, and this temperature is typically fixed at the condensation temperature of the dust species. This condition is not necessarily true for the highly evolved AGB stars as well as post-AGB stars. 2Dust adopts a more general approach (see Ueta \& Meixner 2003) by using the inner radius to calculate the temperature structure in the shell. For sources with detached shells, the inner radius can be directly observed in high-resolution images. The shape of near- and mid-IR SED constrains the inner radius and therefore the temperature at this radius as well. The inner radius of the shell need not, therefore, correspond to the condensation radius, and the resulting temperature may not be equal to the condensation temperature of the dust.

The ratio $R_{\max } / R_{\text {in }}$ of the outer radius of the dust shell to the inner radius is kept fixed at 1000 . The outer radius determines the total shell mass, which in turn is related to the mass-loss timescale. While this timescale is an important quantity, we are more interested in obtaining the AGB mass-loss rate, which is only weakly sensitive to changes in the outer radius as long as it is large enough to include contributions to the flux at the longest wavelengths of interest (in our case, the $24 \mu \mathrm{m}$ band) from the coldest dust in the shell. Like van Loon et al. (2003), we find that the output SED is insensitive to changes in $R_{\max }$ out to $\sim 30 \mu \mathrm{m}$. The mass-loss rate is, however, very sensitive to the value chosen for the inner radius. Observations of Galactic carbon stars (e.g., IRC+10216, Danchi et al. 1990, 1995) and results of radiative transfer modeling (e.g., van Loon et al. 1999; Groenewegen et al. 1998) suggest inner radii in the range $\sim 2-20$ stellar radii $\left(R_{*}\right)$, while simple estimates based on energy balance suggest that amorphous carbon dust should form within a few $R_{*}$ (Höfner 2007). We vary the inner radius within these bounds until the desired SED shape is obtained.

\subsubsection{Dust grain properties}

We model the dust around LPV 28579 using amorphous carbon (optical constants from the "ACAR" sample of Zubko et al. 1996) and silicon carbide (optical constants from Pégourié 1988) grains in order to reproduce the strong $11.3 \mu \mathrm{m} \mathrm{SiC}$ feature and long-wavelength continuum observed.

Our grain sizes are distributed according to the Kim et al. (1994) (KMH) prescription ${ }^{7}$, with a power-law falloff of index $\gamma=3.5$. We vary the minimum grain size $a_{\min }$ and the scale height $a_{0}$ until the shape of the spectrum is reproduced. We find noticeable changes in the output SED only when $a_{\text {min }}$ is increased beyond $\sim 0.1 \mu \mathrm{m}$. This insensitivity to the small grain size limit is due in part to the fact that larger grains contribute more significantly to the dust mass, making it easier to place constraints on the scale height than on the minimum grain size (a similar result was obtained by Speck et al. 2009). Given this distribution of sizes as input, 2Dust then internally calculates absorption and scattering cross-sections and asymmetry factors for an "average" spherical dust grain from the Mie theory (Mie 1908) assuming isotropic scattering. We run the code in the Harrington averaging (Harrington et al. 1988) mode, which gives an average grain size of $\sim 0.1 \mu \mathrm{m}$, the typical value used in single-size models (e.g., van Loon et al. 1999; Groenewegen 2006).

2Dust requires as input the optical depth at a reference wavelength, which we choose to lie near the center of the SiC emission $(11.3 \mu \mathrm{m})$. We iteratively adjust this optical depth and relative abundance of $\mathrm{SiC}$ until the shape of the feature is satisfactorily reproduced.

\subsubsection{Fitting procedure}

2Dust uses the dust opacities, dust grain and shell properties and the density variation as input to first calculate the density $\rho_{\text {in }}$ at the inner radius from the relation

$\tau_{\lambda}=\kappa_{\lambda} \int_{R_{\text {in }}}^{R_{\text {out }}} \rho(r) \mathrm{d} r$

${ }^{7} n(a) \propto a^{-\gamma} \exp \left[-a / a_{0}\right]$ with $a>a_{\min }$ 
where $\tau_{\lambda}$ is the optical depth at the reference wavelength $\lambda$, and $\kappa_{\lambda}$ is the opacity averaged over dust size and composition. The total dust mass in the shell is calculated once $\rho_{\text {in }}$ is known. This is divided by the wind crossing time in the shell to obtain

$\dot{M}=4 \pi \frac{\tau_{\lambda}}{\kappa_{\lambda}} R_{\text {in }} v_{\exp }$.

The dust mass-loss rate can be calculated once a value for the expansion velocity is chosen.

The overall fitting procedure is summarized as follows. Starting with the model photosphere that comes closest in flux to the luminosity of the source (see Sect. 3.1.1) we modify the inner radius, optical depth, $\mathrm{SiC}$ abundance and the range of grain sizes until the observed SED as well as the SiC feature strength is reproduced. In order to compare the results of 2Dust with our observations, we generate synthetic photometry in the $U B V I J H K_{\mathrm{s}}$ as well as IRAC and MIPS24 bands in a manner similar to Srinivasan et al. (2009). At each step, we scale the 2Dust output SED to the Epoch $18 \mu \mathrm{m}$ flux for comparison. The scaling affects the luminosity as well as the mass-loss rate, and in Sect. 3.1.5 we investigate the quality of the fit by varying this scale, which results in a range of predicted luminosities and mass-loss rates.

Starting with the $T_{\text {eff }}=3000 \mathrm{~K}$ photosphere, we first increase the optical depth until we roughly match the optical extinction as well as the mid-IR continuum observed in the photometry. The inner radius is now varied to improve the fit in the near-IR and IRAC part of the SED (the outer radius is kept fixed at $1000 R_{\text {in }}$, see Sect. 3.1.2). At this point, we compare the $\mathrm{SiC}$ features in the model and the IRS spectrum, and change the relative abundance of $\mathrm{SiC}$ in the model if required. In general, this change affects the IRAC fluxes, and we compensate for this by modifying the optical depth and/or inner radius as needed. Finally, we vary the minimum and maximum grain sizes to improve the fit. As noted in Sect. 3.1.3, changes in the minimum grain size do not significantly affect the spectrum. We terminate the iterative procedure described above when we are able to satisfactorily match the optical, near-IR and IRAC/MIPS24 photometry for LPV 28579, as well as reproduce the $11.3 \mu \mathrm{m}$ feature in its IRS spectrum. The resulting best-fit model is described below.

\subsubsection{Dust fit results and sensitivity to parameter variation}

Table 1 summarizes the fixed parameters as well as the values of the free parameters corresponding to the best-fit model. The 2Dust output spectrum is superimposed on the photometry and IRS spectrum in Fig. 3. The luminosity of the model photosphere is lesser than the value calculated for LPV 28579 from photometry, so the entire SED has to be scaled up. The value of this scale factor decides the predicted luminosity and massloss rate, as well as the fit quality. We find that we are able to fit the photometry as well as the IRS spectrum for a scale factor of 1.08, which corresponds to a luminosity of about $6580 L_{\odot}$. Taking into account the photometric errors, a $1 \%$ to $15 \%$ raise in model luminosity (corresponding to luminosities in the range $\left.6150-7010 L_{\odot}\right)$ result in good fits $(\$ 7 \%$ variation around the best-fit value). To demonstrate that this uncertainty is small compared to the difference in luminosities between the two epochs, we scale the best-fit model down to fit the Epoch 2 photometry and find a luminosity of about $4810 L_{\odot}(\sim 27 \%$ fainter $)$. The scaled best-fit dust mass-loss rate for Epoch 1 data is found to be about $2.5 \times 10^{-9} M_{\odot} \mathrm{yr}^{-1}$. The optical depth at $11.3 \mu \mathrm{m}$ is 0.28 .
The inner radius of the dust shell is $4.4 R_{*}\left(1430 R_{\odot}\right)$ and the temperature at this distance from the star is $1310 \mathrm{~K}$.

The results of the model fitting are most sensitive to changes in $R_{\text {in }}$, the optical depth, the $\mathrm{SiC}$ dust abundance and the value of $a_{0}$. When we have created a grid of models, we will be able to derive chi-squared errors for the parameters. For the moment, we determine the acceptable range of variation in the parameters mentioned above as follows. We create boundary ranges on the data by allowing the photometry to change by up to 3 times the uncertainty and by restricting the spectroscopy to a range bounded by its $1 \sigma$ uncertainty, because of lower signal-to-noise ratio associated with the spectra. Using this range of data variation, we determine by eye the range of variation in the model fit due to changes in a certain parameter while keeping all other parameter values fixed at their best-fit model values. The range of parameters thus calculated are shown in Table 1. Figure 4 illustrates this process by showing the range in acceptable model fits as a result of changing the optical depth.

The process described above helps us place a rough constraint on the variation of the important output parameters - the luminosity, mass-loss rate and the dust temperature on the inner radius. We find that changing the inner radius of the dust shell causes the largest variation in both the temperature at the inner radius as well as the mass-loss rate. We find $T_{\text {in }}$ values between $1260 \mathrm{~K}$ and $1380 \mathrm{~K}$. The mass-loss rate is in the range (2.4-2.9) $\times 10^{-9} M_{\odot} \mathrm{yr}^{-1}$. The variation of parameter values thus gives us an estimate of the required data quality if the derived mass-loss rates are to be within $\sim 15 \%$, assuming our value for $v_{\text {exp }}$ is correct.

\subsection{Modeling the circumstellar gas}

The moderate optical depth of the circumstellar shell should be effective in attenuating photospheric features. However, LPV 28579 has strong absorption features around $13.7 \mu \mathrm{m}$. The features therefore originate in the circumstellar region (see, e.g., Zijlstra et al. 2006; Matsuura et al. 2006). In Galactic carbon stars, there is also a contribution to this wavelength range due to $\mathrm{HCN}$, but due to the low abundance of nitrogen, this effect is not pronounced in LMC stars (Matsuura et al. 2006).

We simulate the molecular bands of $\mathrm{CO}$ and $\mathrm{C}_{2} \mathrm{H}_{2}$ in LPV 28579 using the slab model code of Matsuura et al. (2002) with recent line lists published by Rothman et al. (2009) as part of the HITRAN database. Assuming that the majority of the molecular absorption comes from the circumstellar shell, the excitation temperatures are chosen to be cooler than the temperature at the inner shell radius, i.e., $T_{\mathrm{ex}}<T_{\mathrm{in}}$. Based on these considerations, we derive excitation temperatures of $1000 \mathrm{~K}$ for both species, with column densities of $10^{19} \mathrm{~cm}^{-2}$ and $3 \times 10^{21} \mathrm{~cm}^{-2}$ for $\mathrm{C}_{2} \mathrm{H}_{2}$ and $\mathrm{CO}$ respectively. Figure 2 shows the locations of the spectral features for the parameters above. The resulting (gas+dust) fit is in good agreement with the spectrum and is plotted in Fig. 3. The CO band is at the edge of the IRS spectrum so we do not have observational constraints on it, but we are able to reproduce the $13.7 \mu \mathrm{m}$ feature using $\mathrm{C}_{2} \mathrm{H}_{2}$. We note here that the model is useful only for identifying the molecular bands and to roughly estimate the excitation temperatures and densities of these molecular species, it can not be used to determine the details of the atmospheric structure. A determination of the mass-loss rate would also require knowledge of the location of the slab of $\mathrm{C}_{2} \mathrm{H}_{2}$ in the circumstellar envelope. As this quantity is very uncertain, we do not calculate a gas mass-loss rate using this method. 


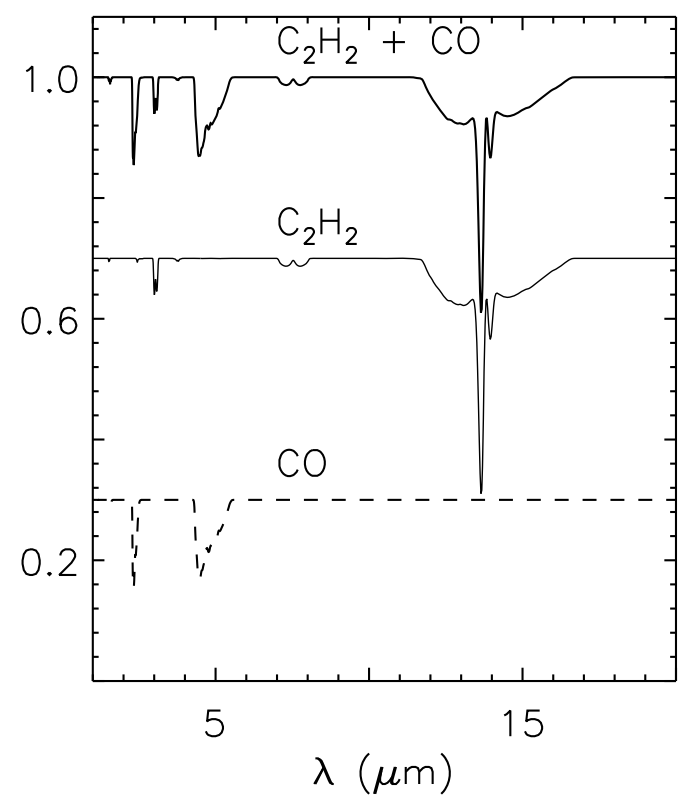

Fig. 2. The synthetic spectra for CO (dashed line, column density = $3 \times 10^{21} \mathrm{~cm}^{-2}$ ) and $\mathrm{C}_{2} \mathrm{H}_{2}$ (thin solid line, column density $=10^{19} \mathrm{~cm}^{-2}$ ) from the HITRAN database and Rothman et al. (2009). The combined spectrum (thick solid line) used to model the molecular contribution to the observed spectrum is also shown. The spectra for $\mathrm{CO}$ and $\mathrm{C}_{2} \mathrm{H}_{2}$ are shifted down for clarity.

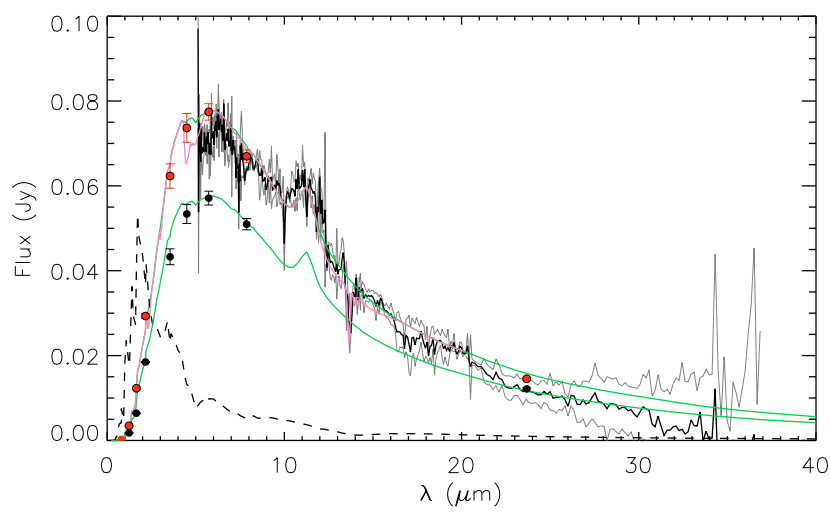

Fig. 3. The results of modeling the dust and gas around LPV 28579. The SAGE Epoch 1 (red dots) and epoch 2 (black dots) photometry as well as SAGE-Spec spectrum (black curve) shown with the bestfit 2Dust RT model (green curves) scaled to fit Epoch 1 and Epoch 2 photometry. The photosphere model corresponding to the best-fit SED is also shown (dashed curve). The model SED is convolved with the synthetic molecular spectrum shown in Fig. 2 to reproduce the $13.7 \mu \mathrm{m}$ feature (pink curve).

\section{Discussion}

The long-term goal of this study is to use 2Dust modeling of carbon star circumstellar shells to fit the photometry of the $\sim 7000$ SAGE C-rich AGB candidates, along with any carbon stars found among the $\sim 1400$ sources in the SAGE extreme AGB list, in order to derive their mass-loss rates and to estimate the total mass-loss return from AGB stars to the LMC ISM. We are also deriving the dust properties for O-rich AGB stars in a parallel study (Sargent et al. 2010, in press). In this section, we weight the simplifying assumptions used in our modeling of LPV 28579 against this future goal. Our study of the variation in model parameters demonstrates that we place reasonable constraints on the inner shell radius, the temperature at this radius, dust grain properties and the resulting mass-loss rates. We discuss the results of our modeling in detail below.

\subsection{Dust properties}

Figure 3 illustrates the good agreement between our model using an $\mathrm{AmC}+\mathrm{SiC}$ dust composition and the overall continuum as well as the $11.3 \mu \mathrm{m}$ feature observed in the spectrum. Our combined gas+dust model improves on the 2Dust prediction by fitting the $13.7 \mu \mathrm{m}$ absorption. We note two minor discrepancies: the silicon carbide feature seems broader than the model prediction, and the model fit systematically overestimates the flux at wavelengths longward of $\sim 20 \mu \mathrm{m}$. A broadening of the $\mathrm{SiC}$ feature may indicate self-absorption (see, e.g., Speck et al. 1997) corresponding to a high optical depth. The long-wavelength disagreement is probably due to the poor background subtraction (see Sect. 2.3) rather than due to a modeling issue. Given the low signal-to-noise of the spectrum, it is not certain if these discrepancies are real; we are therefore unable to justify a detailed refinement of our RT model.

Our best-fit model predicts a temperature of $1310 \mathrm{~K}$ at the inner radius of the dust shell, which is located at $4.4 R_{*}$. In Sect. 3.1.5, we estimate the inner edge to lie within (4.0-4.8) $R_{*}$ (corresponding to temperatures of $1260-1380 \mathrm{~K}$ ), which is consistent with the theoretical predictions of Höfner (2007) for the condensation of amorphous carbon dust $\left(T_{\text {cond }}=1500 \mathrm{~K}\right.$, $\left.R_{\text {in }}=3 R_{*}\right)$. The range of temperatures are slightly warmer than would be expected for LMC carbon stars of comparable massloss rates (see, e.g., Fig. 4 and Eq. (1) of Groenewegen et al. 2009). This may be due to the fact that we are using a warmer central star. Here we are limited by the availability of the colder photospheres of comparable luminosity. Moreover, as already discussed, the moderate optical depth prevents us from using the observed near-IR colors to estimate the central star's effective temperature.

The $\mathrm{KMH}$ grain size distribution for the best-fit model is defined by $\gamma=3.5, a_{\min }=0.01 \mu \mathrm{m}$ and $a_{0}=1.0 \mu \mathrm{m}$. As noted in Sect. 3.1.3, the shape of the output SED is sensitive to the size of the largest grains, but does not vary appreciably with changes in the minimum grain size up $\sim 0.1 \mu \mathrm{m}$. About $15-18 \%$ of the mass is contained in grains with diameters exceeding $1 \mu \mathrm{m}$, depending on the minimum grain size. For comparison, only $0.1 \%$ of the dust mass ejected from IRC+10216 $\left(L \sim 1.5 \times 10^{4} L_{\odot}\right.$, $v_{\text {exp }}=15 \mathrm{~km} \mathrm{~s}^{-1}$, dust MLR $\left.\sim 8 \times 10^{-8} M_{\odot} \mathrm{yr}^{-1}\right)$ is in micronsized particles (Jura 1994).

The SiC content derived from the best-fit model is comparable to values derived for dusty Galactic carbon stars (Groenewegen et al. 1998). The lower metallicity of the LMC would imply a lower silicon abundance in general, and Groenewegen et al. (2007) find lower SiC/AmC ratios for the LMC compared to Galactic stars. By studying the effect of varying the $\mathrm{SiC}$ abundance on the output SED, we note that the SiC content can be in the range 10-16\%. In this paper, we assume a gas:dust ratio $\Psi=200$. This value was determined for carbon stars in the solar neighbourhood (Jura 1986). An accurate determination of the gas:dust ratio is still to be made for LMC carbon stars. Some observational and theoretical studies (e.g., Matsuura et al. 2005; Wachter et al. 2008) indicate that the carbon star gas:dust ratio may not be metallicity dependent. In fact, recent 


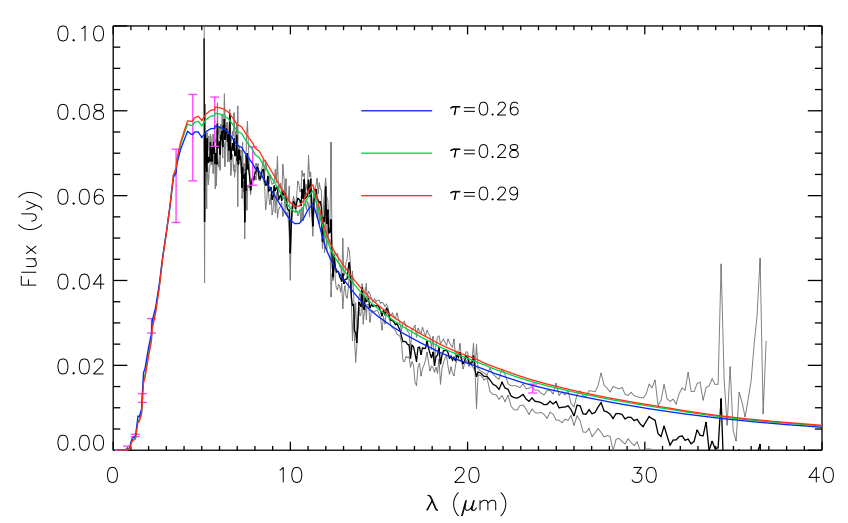

Fig. 4. The sensitivity of the 2Dust model output to optical depth. The SAGE Epoch 1 photometry for LPV 28579 is allowed a $3 \sigma$ variation (magenta error bars), and the spectroscopy is kept to within $1 \sigma$ (gray curves). Model SEDs for $11.3 \mu \mathrm{m}$ optical depth values spanning this range of allowed fluxes are also shown. The optical depth for good fits ranges from 0.26 (blue curve) to 0.29 (red curve). The best-fit curve for $\tau=0.28$ (green) is also shown for comparison.

studies (Groenewegen et al. 2007; Wachter et al. 2008; Mattsson et al. 2008) have found that the mass-loss rates of carbon stars may not be metallicity dependent. The gas:dust ratio can be estimated from the mass fraction of $\mathrm{SiC}$ in the dust if we also know the fraction of the total Si mass that is in the dust. Assuming that the $\mathrm{Si}$ abundance scales with metallicity (The LMC silicon abundance is not well constrained; see the discussion in Matsuura et al. 2005) we obtain $\Psi$ in the range 200-570. However, we have assumed here that all of the Si goes into the dust. In reality, the fraction of $\mathrm{Si}$ in dust depends indirectly on the amount of carbon in the dust. For example, if half of the carbon remaining after $\mathrm{CO}$ formation goes into dust, then $8-13 \%$ of the $\mathrm{Si}$ is in dust ${ }^{8}$. The situation improves for higher $\mathrm{C} / \mathrm{O}$ ratio - for $\mathrm{C} / \mathrm{O}=1.8$, the $\mathrm{Si}$ dust fraction is $20-34 \%$. An alternative way to estimate the gas:dust ratio is to use the van Loon (2000) relationship $\Psi \sim Z^{-1 \pm 0.3}$ for carbon stars, which corresponds to $\Psi=300-1000$ for the LMC. Due to the lack of constraints on the various quantities that determine the gas:dust ratio, we use $\Psi=200$ when quoting a total mass-loss rate for LPV 28579 , noting that the mass-loss rate could be up to about 5 times larger based on the calculations above.

\subsection{Mass-loss rate}

Our 2Dust model predicts a dust mass-loss rate of $2.5 \times$ $10^{-9} M_{\odot} \mathrm{yr}^{-1}$. For a gas:dust ratio of 200 , the total mass-loss rate is then $5 \times 10^{-7} M_{\odot} \mathrm{yr}^{-1}$. This rate is on the low end of the van Loon et al. (1999) mass-loss rates for the brightest, most obscured LMC carbon stars as well as the rates calculated for the Groenewegen et al. (2009) sample. The mass-loss rates calculated from the [3.6]-[8.0] and $K-[8.0]$ colors of LPV 28579 using equations 1 and 2 of Matsuura et al. (2009) are slightly higher $\left(1.4 \times 10^{-6} M_{\odot} \mathrm{yr}^{-1}\right.$ and $2.5 \times 10^{-6} M_{\odot} \mathrm{yr}^{-1}$ respectively $)$. However, our dust MLR is consistent with the value determined from the $8 \mu \mathrm{m}$ flux - using the excess-MLR relation for extreme AGB stars from Paper I, we get a rate of $3.9 \times 10^{-9} M_{\odot} \mathrm{yr}^{-1}$.

\footnotetext{
${ }^{8}$ Here, we have assumed that carbon and silicon are the only constituents of the dust. A more realistic estimate would include metallic iron dust, which somewhat counteracts the low Si fraction and lowers the gas:dust ratio.
}

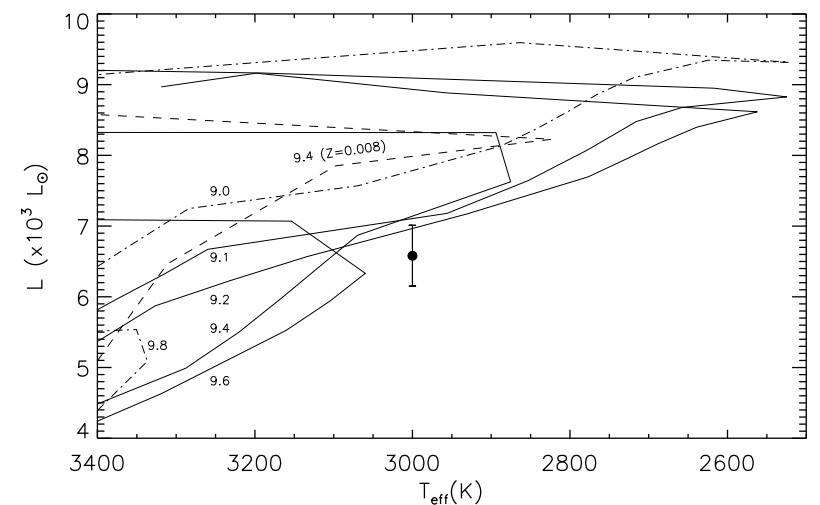

Fig. 5. LPV 28579 (black dot with error bar representing range of calculated Epoch 1 luminosities) on the $L-T_{\text {eff }}$ diagram. The solid and dotdashed lines, tagged according to their age $(\log t / \mathrm{Gyr})$, are the Marigo et al. (2008) isochrones for $Z=0.006$. For comparison, the $Z=0.008$ track for $\log t / \mathrm{Gyr}=9.4$ is also shown (dashed line).

Our total rate agrees with the values calculated using the periodMLR relations of Vassiliadis \& Wood (1993) and Groenewegen et al. (1998) $\left(10^{-7} M_{\odot} \mathrm{yr}^{-1}\right.$ and $7.4 \times 10^{-7} M_{\odot} \mathrm{yr}^{-1}$ respectively, for a period of $356.2 \mathrm{~d})$. The value obtained for the mass-loss rate is sensitive to the $v_{\text {exp }}$ adopted (see Eq. (2)). The outflow velocities for Galactic carbon stars are typically $10 \mathrm{~km} \mathrm{~s}^{-1}$, but can be up to about twice as high. The Wachter et al. (2008) models predict LMC outflow velocities to be about half that of comparable Galactic sources. This range of velocities introduces an uncertainty of a factor of two in the calculated mass-loss rate.

\subsection{Evolutionary state}

In order to understand our $L$ and $T_{\text {eff }}$ values for LPV 28579 in the context of its evolutionary state, we identify its location in the Hertzsprung-Russel (HR) diagram in relation to the Marigo et al. (2008) theoretical isochrones. We choose a metallicity of $Z=0.006$, which is consistent with observational estimates for the metallicity of the LMC. We generate these isochrones using their CMD interactive web interface ${ }^{9}$ so that we can also extract the initial stellar masses for the stellar tracks. Figure 5 shows the location of LPV 28579 on the HR diagram. Also shown are selected isochrones for $Z=0.006$ (ages ranging from $\log t / \mathrm{Gyr}=9.0$ to 9.8 ). We find that the $\log t / \mathrm{Gyr}=9.1$ to 9.6 isochrones are able to produce the range of luminosities we have estimated for LPV 28579 in this study, which gives us an age in the range 1.6-4 Gyr. As noted by Marigo et al. (2008), this is the typical age of the O-rich AGB population that undergoes a smooth transition to C-rich chemistry due to third dredge-up (also see the discussion of the multiple O-rich populations in Paper I). The initial masses corresponding to these isochrones were in the range 1.3-2 $M_{\odot}$. Figure 3 of Groenewegen \& de Jong (1994) shows the evolution of 1.25 and $5 M_{\odot}$ AGB stars in period-luminosity space. Based on its luminosity and primary period, LPV 28579 falls slightly upward of the $1.25 M_{\odot}$ track, providing a mass comparable with our estimate using theoretical isochrones.

The derived age (and hence progenitor mass) range is dependent on the metallicity of the isochrones as well as our $T_{\text {eff }}$ estimate for LPV 28579. Figure 5 demonstrates the effect

\footnotetext{
${ }^{9}$ http://stev.oapd.inaf.it/cmd
} 
of changing the metallicity (compare the $\log t / \mathrm{Gyr}=0.94$ isochrones for $Z=0.006$ and 0.008 ). At lower metallicities, the isochrones predict carbon stars at lower luminosities. The optically thick shell of LPV 28579 makes the precise determination of $T_{\text {eff }}$ difficult (see 3.1.1) so that our choice of model photosphere is made on the basis of the luminosity estimated from photometry. The range of ages and masses from isochrone comparison is dependent on how well we can constrain the effective temperature. If we assume that $T_{\text {eff }}$ is cooler than $3100 \mathrm{~K}$, we obtain the age and mass range above. For $T_{\text {eff }}$ under $3050 \mathrm{~K}$, the range reduces to $\log t / \mathrm{Gyr}=9.2-9.5$ giving masses in the range 1.4-1.7 $M_{\odot}$.

The total mass-loss rate of $5.0 \times 10^{-7} M_{\odot} \mathrm{yr}^{-1}$ is comparable to the nuclear-burning rate at the luminosity of the star (see Fig. 9 in van Loon et al. 1999), which suggests that LPV 28579 may undergo the superwind phase at the end of its life on the AGB.

\section{Conclusions}

We have modeled the circumstellar shell of the carbon-rich asymptotic giant branch star LPV 28579 using the radiative transfer code 2Dust to fit the SAGE photometry and SAGESpec spectroscopy available for the source. Using a mixture of amorphous carbon and silicon carbide dust with $12 \% \mathrm{SiC}$ by mass, and dust grains ranging in size from $0.01 \mu \mathrm{m}$ to $\sim 1.0 \mu \mathrm{m}$, we find a luminosity of $6580 L_{\odot}$ and a dust mass-loss rate of $2.5 \times 10^{-9} M_{\odot} \mathrm{yr}^{-1}$. The inner radius of the shell is at 4.4 times the stellar radius and at a temperature of $1310 \mathrm{~K}$. The optical depth at $11.3 \mu \mathrm{m}$ is found to be 0.28 . We model the molecular features in the circumstellar shell with slab models of $\mathrm{C}_{2} \mathrm{H}_{2}$ and $\mathrm{CO}$, and calculate column densities of $10^{19} \mathrm{~cm}^{-2}$ and $3 \times 10^{21} \mathrm{~cm}^{2}$ for the two species respectively. The model SED when combined with the gas model shows excellent agreement with the observed spectrum and photometry. The total mass-loss rate assuming a gas:dust ratio of 200 is comparable to estimates derived from empirical relations involving the period, color and mid-infrared flux. LPV 28579 has properties typical of dust-enshrouded LMC carbon Miras, and the model derived in this work defines a baseline set of carbon star dust properties in our upcoming model grid.

Acknowledgements. The authors would like to thank the anonymous referee for their helpful comments on the manuscript. This work is based on observations made with the Spitzer Space Telescope, which is operated by the Jet Propulsion Laboratory, California Institute of Technology under NASA contract 1407. The research in this paper has been funded by Spitzer grant 1310534 and NASA grant NAG5-12595. The authors have made use of the SIMBAD astronomical database and would like to thank those responsible for its upkeep. The authors also thank Bernie Shiao at STScI for his hard work on the SAGE database and his kind assistance.

\section{References}

Blanco, A., Borghesi, A., Fonti, S., \& Orofino, V. 1998, A\&A, 330, 505 Blum, R. D., Mould, J. R., Olsen, K. A., et al. 2006, AJ, 132, 2034 Bressan, A., Granato, G. L., \& Silva, L. 1998, A\&A, 332, 135 Chan, S. J., \& Kwok, S. 1990, A\&A, 237, 354

Cioni, M.-R. L., Girardi, L., Marigo, P., \& Habing, H. J. 2006, A\&A, 448, 77

Cutri, R. M., et al. 2003, The IRSA 2MASS All-Sky Point Source Catalog, NASA IPAC Infrared Science Archive, available at http://irsa.ipac. caltech.edu/applications/Gator

Danchi, W. C., Bester, M., Degiacomi, C. G., McCullough, P. R., \& Townes, C. H. 1990, ApJ, 359, L59

Danchi, W. C., Bester, M., Greenhill, L. J., et al. 1995, Ap\&SS, 224, 447

Dwek, E. 1998, ApJ, 501, 643
Epchtein, N., de Batz, B., Copet, E., et al. 1994, Ap\&SS, 217, 3 Epchtein, N., Deul, E., Derriere, S., et al. 1999, A\&A, 349, 236 Fazio, G. G., Stier, M. T., Wright, E. L., \& McBreen, B. 1980, ApJ, 237, L39

Fazio, G. G., Hora, J. L., Allen, L. E., et al. 2004, ApJS, 154, 10

Feast, M. 1999, New Views of the Magellanic Clouds, 190, 542

Fraser, O. J., Hawley, S. L., \& Cook, K. H. 2008, AJ, 136, 1242

Gautschy-Loidl, R., Höfner, S., Jørgensen, U. G., \& Hron, J. 2004, A\&A, 422, 289

Gehrz, R. D., et al. 2007, Review of Scientific Instruments, 78, 011302

Groenewegen, M. A. T. 1995, A\&A, 293, 463

Groenewegen, M. A. T. 2004, A\&A, 425, 595

Groenewegen, M. A. T. 2006, A\&A, 448, 181

Groenewegen, M. A. T., \& de Jong, T. 1994, A\&A, 288, 782

Groenewegen, M. A. T., Whitelock, P. A., Smith, C. H., \& Kerschbaum, F. 1998, MNRAS, 293, 18

Groenewegen, M. A. T., Sevenster, M., Spoon, H. W. W., \& Pérez, I. 2002, A\&A, 390,511

Groenewegen, M. A. T., Wood, P. R., Sloan, G. C., et al. 2007, MNRAS, 376, 313

Groenewegen, M. A. T., Sloan, G. C., Soszyński, I., \& Petersen, E. A. 2009, A\&A, 506, 1277

Habing, H. J. 1996, A\&ARv, 7, 97

Harrington, J. P., Monk, D. J., \& Clegg, R. E. S. 1988, MNRAS, 231, 577

Höfner, S. 2007, in Why Galaxies Care About AGB Stars: Their Importance as Actors and Probes, ASP Conf. Ser., 378, 145

Hrivnak, B. J., Volk, K., \& Kwok, S. 2009, ApJ, 694, 1147

Iben, I., Jr., \& Renzini, A. 1983, ARA\&A, 21, 271

Ita, Y., Tanabé, T., Matsunaga, N., et al. 2004, MNRAS, 353, 705

Jones, T. W., \& Merrill, K. M. 1976, ApJ, 209, 509

Jura, M. 1986, ApJ, 303, 327

Jura, M. 1994, ApJ, 434, 713

Kato, D., Nagashima, C., Nagayama, T., et al. 2007, PASJ, 59, 615

Kemper, F., Woods, P. M., Antoniou, V., et al. 2010, PASP, 122, 683

Kim, S.-H., Martin, P. G., \& Hendry, P. D. 1994, ApJ, 422, 164

Lagadec, E., Zijlstra, A. A., Sloan, G. C., et al. 2007, MNRAS, 376, 1270

Lagadec, E., Zijlstra, A. A., Mauron, N., et al. 2010, MNRAS, 403, 1331

Lorenz-Martins, S., \& Lefevre, J. 1993, A\&A, 280, 567

Lorenz-Martins, S., \& Lefevre, J. 1994, A\&A, 291, 831

Loup, C., Forveille, T., Omont, A., Paul, J. F. 1993, A\&AS, 99, 291

Marigo, P., Girardi, L., Bressan, A., et al. 2008, A\&A, 482, 883

Martin, P. G., \& Rogers, C. 1987, ApJ, 322, 374

Matsuura, M., Yamamura, I., Cami, J., Onaka, T., \& Murakami, H. 2002, A\&A, 383,972

Matsuura, M., Zijlstra, A. A., van Loon, J. Th., et al. 2005, A\&A, 434, 691

Matsuura, M., Wood, P. R., Sloan, G. C., et al. 2006, MNRAS, 371, 415

Matsuura, M., Zijlstra, A. A., Bernard-Salas, J., et al. 2007, MNRAS, 382, 1889

Matsuura, M., Barlow, M. J., Zijlstra, A. A., et al. 2009, MNRAS, 396, 918

Mattsson, L., Wahlin, R., Höfner, S., \& Eriksson, K. 2008, A\&A, 484, L5

Meixner, M., Zalucha, A., Ueta, T., Fong, D., \& Justtanont, K. 2004, ApJ, 614, 371

Meixner, M., Gordon, K. D., Indebetouw, R., et al. 2006, AJ, 132, 2268

Mie, G., 1908, Ann. Phys., 330, 377

Nagayama T., Nagashima, C., Nakajima, Y., et al. 2003, in Instrument Design and Performance for Optical/Infrared Ground-based Telescopes, ed. M. Iye, \& A. F. M. Moorwood, SPIE, 4841, 459

Nenkova, M., Ivezić, Ž., \& Elitzur, M. 2000, in Thermal Emission Spectroscopy and Analysis of Dust, Disks, and Regoliths, ASP Conf. Ser., 196, 77

Neugebauer, G., \& Leighton, R. B. 1969, NASA SP, Washington: NASA

Olofsson, H., Eriksson, K., Gustafsson, B., \& Carlstrom, U. 1993, ApJS, 87, 267

Pégourié, B. 1988, A\&A, 194, 335

Pitman, K. M., Hofmeister, A. M., Corman, A. B., \& Speck, A. K. 2008, A\&A, 483, 661

Rieke, G. H., Young, E. T., Engelbracht, C. W., et al. 2004, ApJS, 154, 25

Rowan-Robinson, M., \& Harris, S. 1983, MNRAS, 202, 797

Rothman, L. S., Gordon, I. E., Barbe, A., et al. 2009, J. Quant. Spec. Radiat. Transf., 110, 533

Skinner, C. J., \& Whitmore, B. 1988, MNRAS, 234, 79P

Skrutskie, M. F., Cutri, R. M., Stiening, R., et al. 2006, AJ, 131, 1163

Sloan, G. C., Kraemer, K. E., Matsuura, M., et al. 2006, ApJ, 645, 1118

Sloan, G. C., Kraemer, K. E., Wood, P. R., et al. 2008, ApJ, 686, 1056

Soszyñski, I., Udalski, A., Szymañski, M. K., et al. 2009, Acta Astron., 59, 239

Speck, A. K., Barlow, M. J., \& Skinner, C. J. 1997, Meteoritics and Planetary Science, 32, 703

Speck, A. K., Corman, A. B., Wakeman, K., Wheeler, C. H., \& Thompson, G. 2009, ApJ, 691, 1202

Srinivasan, S., Meixner, M., Leitherer, C., et al. 2009, AJ, 137, 4810 
Stanghellini, L., Shaw, R. A., \& Gilmore, D. 2005, ApJ, 622, 294

Suh, K.-W. 2000, MNRAS, 315, 740

Szymański, M. K. 2005, Acta Astron., 55, 43

Ueta, T., \& Meixner, M. 2003, ApJ, 586, 1338

van Leeuwen, F., Feast, M. W., Whitelock, P. A., \& Laney, C. D. 2007, MNRAS, 379,723

van Loon, J. T. 2000, A\&A, 354, 125

van Loon, J. T., Groenewegen, M. A. T., de Koter, A., et al. 1999, A\&A, 351, 559

van Loon, J. T., Marshall, J. R., Matsuura, M., \& Zijlstra, A. A. 2003, MNRAS, 341,1205

Vassiliadis, E., \& Wood, P. R. 1993, ApJ, 413, 641

Vijh, U. P., Meixner, M., Babler, B., et al. 2009, AJ, 137, 3139

Wachter, A., Winters, J. M., Schröder, K.-P., \& Sedlmayr, E. 2008, A\&A, 486, 497

Werner, M. W., Roellig, T. L., Low, F. J., et al. 2004, ApJS, 154, 1
Westerlund, B. E. 1997, Cambridge Astrophys. Ser., 29

Whitelock, P. A., Feast, M. W., van Loon, J. T., \& Zijlstra, A. A. 2003, MNRAS, 342,86

Whitelock, P. A., Feast, M. W., Marang, F., \& Groenewegen, M. A. T. 2006, MNRAS, 369, 751

Wood, P. R., Alcock, C., Allsman, R. A., et al. 1999, Asymptotic Giant Branch Stars, 191, 151

Woods, P. M., Sloan, G. C., Gordon, K. D., et al. 2009, Sage-spectroscopy: The Life-cycle of Dust and Gas in The Large Magellanic Cloud, Data delivery document v1.0, http://data.spitzer.caltech.edu/popular/ sage-spec $/ 20090730$

Zaritsky, D., Harris, J., \& Thompson, I. 1997, AJ, 114, 1002

Zebrun, K., Soszynski, I., Wozniak, P. R., et al. 2001, Acta Astron., 51, 317

Zijlstra, A. A., Matsuura, M., Wood, P. R., et al. 2006, MNRAS, 370, 1961

Zubko, V. G., Mennella, V., Colangeli, L., \& Bussoletti, E. 1996, MNRAS, 282, 1321 